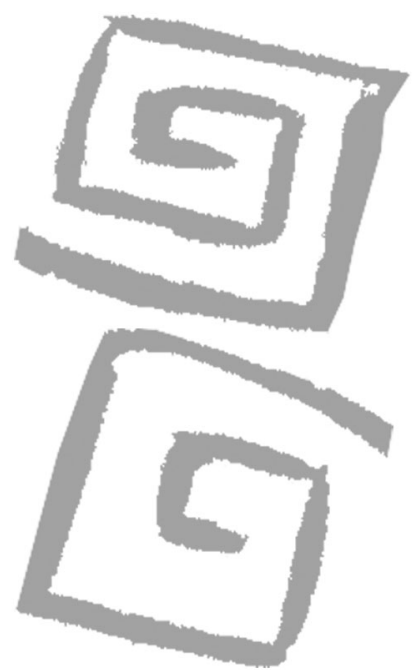

\title{
Gobernanza sistémica para un enfoque de derechos en salud. Un análisis a partir del caso chileno
}

\author{
Systemic governance for a human rights approach to \\ health. An analysis based on the Chilean case
}

Cunill Grau, Nuria; Fernández, María Margarita²; Vergara, Marcos ${ }^{3}$

${ }^{1}$ Administradora Pública. Doctora en Ciencias Sociales. Profesora Titular, Centro de Investigación Sociedad y Políticas Públicas, Universidad de Los Lagos. Profesora Asociada, Escuela de Salud Pública, Universidad de Chile. Asesora Especial, Centro Latinoamericano de Administración para el Desarrollo (CLAD). Chile. Editora en Jefe, Revista del CLAD Reforma y Democracia. nuriacunill@vtr.net.

${ }^{2}$ Nutricionista. Magíster en Ciencias Biológicas. Profesora Asociada, Centro de Investigación Sociedad y Políticas Públicas, Universidad de Los Lagos. Chile. margarita.fernandez@ulagos.cl

${ }^{3}$ Médico. Profesor Asistente, Escuela de Salud Pública, Universidad de Chile. mvergara@med.uchile.cl
RESUMEN El artículo analiza los nudos y desafíos organizacionales, de gestión e institucionales de la construcción de gobernanza sistémica en la implantación de políticas con enfoque de derechos. Adopta como caso la reforma sanitaria chilena iniciada bajo ese enfoque en el año 2004. La metodología integró un análisis cualitativo de normas legales, de instrumentos de gestión y de 40 entrevistas en profundidad efectuadas en 2009 a directivos de salud de distintos establecimientos en tres regiones de Chile. Los hallazgos muestran que en la reforma prevalece una conexión entre incentivos, cálculos privados de beneficios personales y mediciones que no favorece la gestión de las interdependencias y tiende a debilitar los valores de ayuda y cooperación mutua necesarios para alcanzar la integralidad en la solución de las problemáticas que los derechos interpelan. Se concluye, entre otros aspectos, que una aceptación acrítica de los modos de gestión y organización heredados de reformas institucionales previas, genera contradicciones con la necesidad de construir gobernanza para un enfoque de derechos.

PALABRAS CLAVE Reforma Sanitaria; Derechos Humanos; Gerencia/gestión administrativa; Políticas Públicas; Chile.

ABSTRACT The article analyzes organizational, managerial, and institutional chaIlenges in the development of systemic governance for the implementation of policies with a human rights approach, taking as a case study the Chilean health reform initiated with that approach in 2004. The study integrates a qualitative analysis of legal norms, of managerial instruments and of 40 interviews conducted in 2009 with health executives in three regions of Chile. The findings show that in this reform there is a close connection between incentives and calculations of personal benefit that does not favor agency interdependence. The prevalence of this trait tends to undermine the values of mutual aid and cooperation needed to achieve the integral solutions to social problems that a human rights approach demands. The conclusions reached state, in part, that an uncritical acceptance of management and organization methods inherited from previous institutional reforms often creates contradictions in the development of a governance structure conducive to a human rights approach.

KEY WORDS Health Care Reform; Human Rights; Management/organization \& administration; Public Policies, Chile. 


\section{INTRODUCCIÓN}

A diferencia de los modelos instaurados en la mayoría de los países europeos, los sistemas de salud latinoamericanos, en gran parte de los países, se orientaron hacia estratos específicos de la población, lo que produjo un fenómeno de segmentación poblacional y, consiguientemente, la estratificación del ejercicio del derecho a la salud (1). En ese contexto, las reformas institucionales acaecidas en los sectores públicos desde hace casi 30 años, en particular en el caso chileno, han tendido a reforzar la fragmentación de los sistemas de salud al establecer la separación de las funciones de aseguramiento, financiamiento y prestación de los servicios, la ampliación de la participación del sector privado en la provisión de servicios y la descentralización de la atención primaria a las municipalidades, todas ellas medidas guiadas por la premisa de que la creación de competencia para la consecución de financiamiento y su vinculación a "resultados" constituye el incentivo institucional por excelencia a recrear en el sector público.

En paralelo, en la última década adquieren una renovada importancia las políticas públicas con enfoque de derechos (PPED) (2-6), que no solo ponen el acento en la universalidad, la no discriminación y la exigibilidad de los derechos sociales sino también en la importancia de un enfoque holístico o integral en el disfrute de un derecho, lo que obliga a considerar los atributos que crean gobernanza en la institucionalidad a cargo de la implementación de una PPED.

La reforma sanitaria chilena, iniciada en el año 2004 (a), incluyó un componente central de garantización progresiva de derechos exigibles en salud -garantías explícitas de salud, (GES)-, tanto para el sistema previsional (asegurador) público como para el privado, que abarcan derechos de cobertura, plazos máximos para el otorgamiento de las prestaciones, protección financiera y un estándar de calidad para la atención de un conjunto de problemas de salud graves, caros y frecuentes, que representan una alta proporción de la carga de enfermedad y que a la fecha suman 66. A la par, la reforma buscó en el sistema público la adecuación del modelo de atención del primer nivel hacia un modelo de salud familiar. Por otra parte, el reordenamiento institucional provocado por el marco legal de la reforma, retiró las funciones de Autoridad Sanitaria a los 29 servicios de salud -quienes a partir de ello se orientan exclusivamente a la gestión de la red asistencial para la prestación de servicios de salud de su territorio- y las radicó en las 15 Secretarías Regionales Ministeriales de Salud (Seremis). A nivel ministerial, esta separación de funciones se expresó en la creación de dos subsecretarías (o viceministerios), una de Redes Asistenciales y otra de Salud Pública. Las Figuras 1 y 2 dan cuenta de la conformación del sector salud antes y después de la reforma. A partir de allí, puede constatarse tanto la creación de nuevos actores como una complejización de las relaciones.

El argumento acá desarrollado es que, no obstante basarse en el enfoque de derechos, la implementación de la reforma sanitaria chilena ha adoptado los arreglos organizativos y de gestión que son tributarios del patrón de cambios institucionales hegemónico y, por tanto, que no necesariamente resultan consistentes con las exigencias que impone el marco normativo de las PPED en relación a la gobernanza. De esta manera, este artículo busca aportar una perspectiva de análisis de los problemas de implementación complementaria a las tradicionales, que suelen poner énfasis solo en las cuestiones de financiamiento $(7,8)$ o en el análisis de la economía política de los cambios $(9,10)$ (intereses en conflicto, estrategias de resistencias de los posibles actores perdedores, consistencia de la coalición gubernamental, etc.) o en los déficits técnicos, mas no en el hecho de que los arreglos organizativos y de gestión no sean políticamente inocuos, en tanto contribuyen a la creación de reglas de juego que afectan los balances de poder y los valores y, con ello, la construcción de gobernanza.

\section{LA BASE CONCEPTUAL: LA CUESTIÓN DE LA GOBERNANZA SISTÉMICA EN EL ENFOQUE DE DERECHOS}

Según se ha observado (11), la noción de gobernanza ilumina acerca de los límites del gobierno a través de un solo actor y enfatiza en 
que la calidad de las interacciones que genera un determinado arreglo institucional es tan importante como la eficiencia. En el caso de la implementación de las políticas con enfoque de derechos, el acento en la búsqueda de soluciones integrales compromete específicamente a los diversos actores públicos concernidos con la problemática de la salud, tornando a la gobernanza en un atributo necesario que puede complementar nociones como la de las Redes Integradas de Servicios de Salud (RISS) (1). No ocurre lo mismo respecto del sector privado el que, aun implicado en la ejecución del enfoque de derechos, opera bajo relaciones de compra-venta de servicios tanto entre sí (compra) como con el sector público (básicamente venta) (b).

La noción de gobernanza sistémica $(11,13)$, a diferencia del uso que otros autores le han dado (14), remite al alineamiento e integración de los actores públicos para la efectiva protección de un derecho instituido. Bajo esta perspectiva, existe gobernanza sistémica cuando al menos los actores gubernamentales involucrados en el ejercicio de un derecho ciudadano, mantienen relaciones orgánicas y sostenidas de cooperación para producir soluciones integrales a las problemáticas que el derecho interpela.

A partir de diversos aportes que hace la literatura especializada, se asume que al menos tres dimensiones requieren ser atendidas a fin de lograr que la gobernanza sistémica se constituya en un atributo de la institucionalidad pública. Ellas son: la instrumental, la espacial y la valórica.

El argumento es que las relaciones se construyen (o se obstaculizan) a través de estas tres dimensiones que, a la vez, interactúan entre sí, modelándose unas a otras. Ellas, por tanto, definen implícitamente una estrategia de gobernanza, dándole a un conjunto direccionalidad y consistencia en torno a ciertos propósitos.

La dimensión espacial considera que la cooperación basada en la confianza es más eficaz que la basada en la autoridad y remarca la importancia que reviste generar espacios de comunicación recíproca entre la diversidad de actores que tienen incidencia en la ejecución de las PPED, de modo de desencadenar procesos de producción de significados y saberes compartidos $(15,16)$, y aumentar la percepción de su interdependencia. Se sustenta también en la ya amplia literatura que subraya la necesidad de desarrollar procesos deliberativos para aumentar la comprensión mutua y ayudar a lidiar con las diferencias de intereses y perspectivas, que son particularmente significativas en el ámbito público (17-20). Encuentra además un fundamento en los estudios acerca de la gestión de la intersectorialidad, los cuales sugieren que tales espacios son especialmente importantes cuando está planteada la ejecución de políticas públicas sobre problemas sociales multidimensionales que exigen no solo una adecuada coordinación entre varios sectores gubernamentales sino la existencia de visiones compartidas entre ellos y la articulación de sus recursos y saberes $(21,22)$.

La dimensión instrumental alude a la potencialidad de los instrumentos de planificación, presupuestación y evaluación para promover las articulaciones necesarias entre los actores, de modo de hacer viable el derecho instituido. El punto es que los espacios de comunicación recíproca no pueden producir integración si es que los instrumentos de gestión no son integradores. El ejemplo más claro es la ineficacia que tienen, en general, los espacios de coordinación interinstitucional que operan sobre procesos de planificación y presupuestación unilaterales. La importancia de estos aspectos ha sido resaltada indirectamente por la literatura especializada relativa a la administración relacional y a las redes (23-25), así como por la más reciente concerniente a la salud $(1,26)$.

La dimensión valórica subyace a las anteriores. En especial pone la atención en la consistencia entre las metas de resultados inducidas por los instrumentos de gestión y el valor de la cooperación mutua, habida cuenta de la interdependencia que existe entre los valores y las instituciones (27-29).

Con la atención en estas dimensiones lo que queda sugerido es que la existencia de gobernanza sistémica requiere de la construcción de incentivos institucionales para gestionar el cambio hacia una gestión integrada y deliberativa al interior del respectivo sector gubernamental y con los otros sectores que tienen injerencia en los resultados de una PPED (incluidos, naturalmente, a los financieros). Ello es particularmente atingente al sector salud, dada la fragmentación del sistema. 
Figura 1. Red de relaciones del sector salud antes de la reforma sanitaria chilena del año 2004.

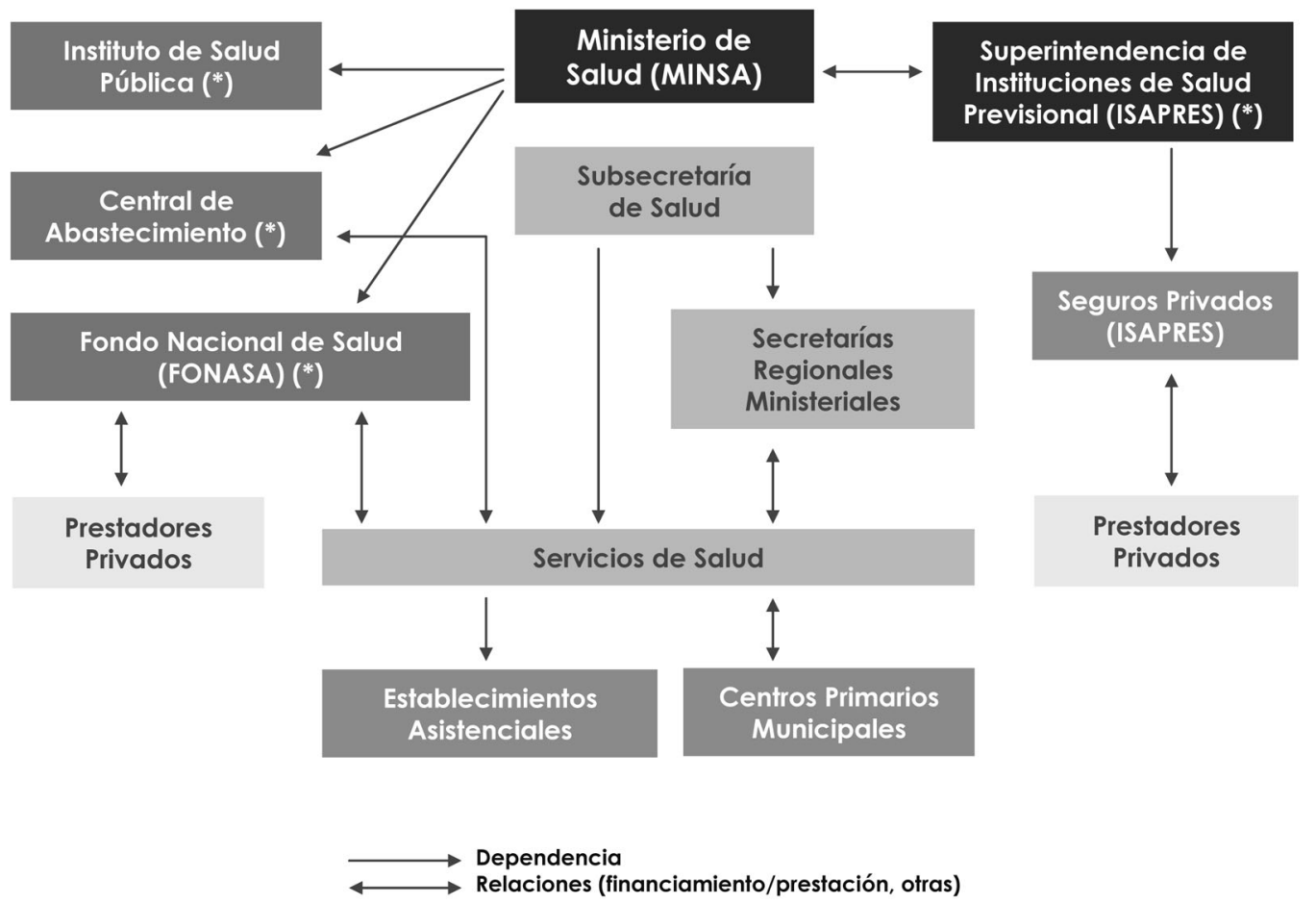

(*) Desconcentrados del Ministerio de Salud, pero regidos por éste.

Fuente: Elaboración propia.

Las expresiones prácticas de la gobernanza sistémica son, al menos, las siguientes: a) espacios institucionalizados de comunicación recíproca (deliberación) entre los actores, a los efectos de garantizar la integridad; b) instrumentos y procesos de gestión en red, que apunten a un manejo integrado de las PPED tanto intra como intersectoriales; y c) criterios de valor coherentes con los procesos de presupuestación, programación y evaluación en red y, en general, con la producción de gobernanza.

\section{MATERIAL Y MÉTODO}

Adoptando el referido marco conceptual, la investigación adoptó como unidad de análisis las relaciones que se promueven al interior de la cadena del sistema público de salud chileno para la implementación de la reforma sanitaria. El estudio, de tipo cualitativo, efectuó un análisis documental de fuentes primarias y secundarias con el propósito de identificar, por una parte, los instrumentos de gestión (planificación, presupuestación, coordinación y evaluación) que responsabilizan a los distintos actores del sector salud por el cumplimiento de metas de resultado en pos de la ejecución de la política de salud. Estos fueron clasificados atendiendo tres criterios:

1) la naturaleza, según la direccionalidad de las acciones de salud: prestación de salud o promoción y prevención;

2) el origen del instrumento: externos al sector salud o propios;

3) el alcance: instrumentos transversales externos que abarcan todo el sector público, instrumentos transversales internos referidos al sector salud en su conjunto o instrumentos específicos a la operación de un ámbito determinado. 
Figura 2. Red de relaciones y funciones del sector salud después de la reforma sanitaria chilena del año 2004.

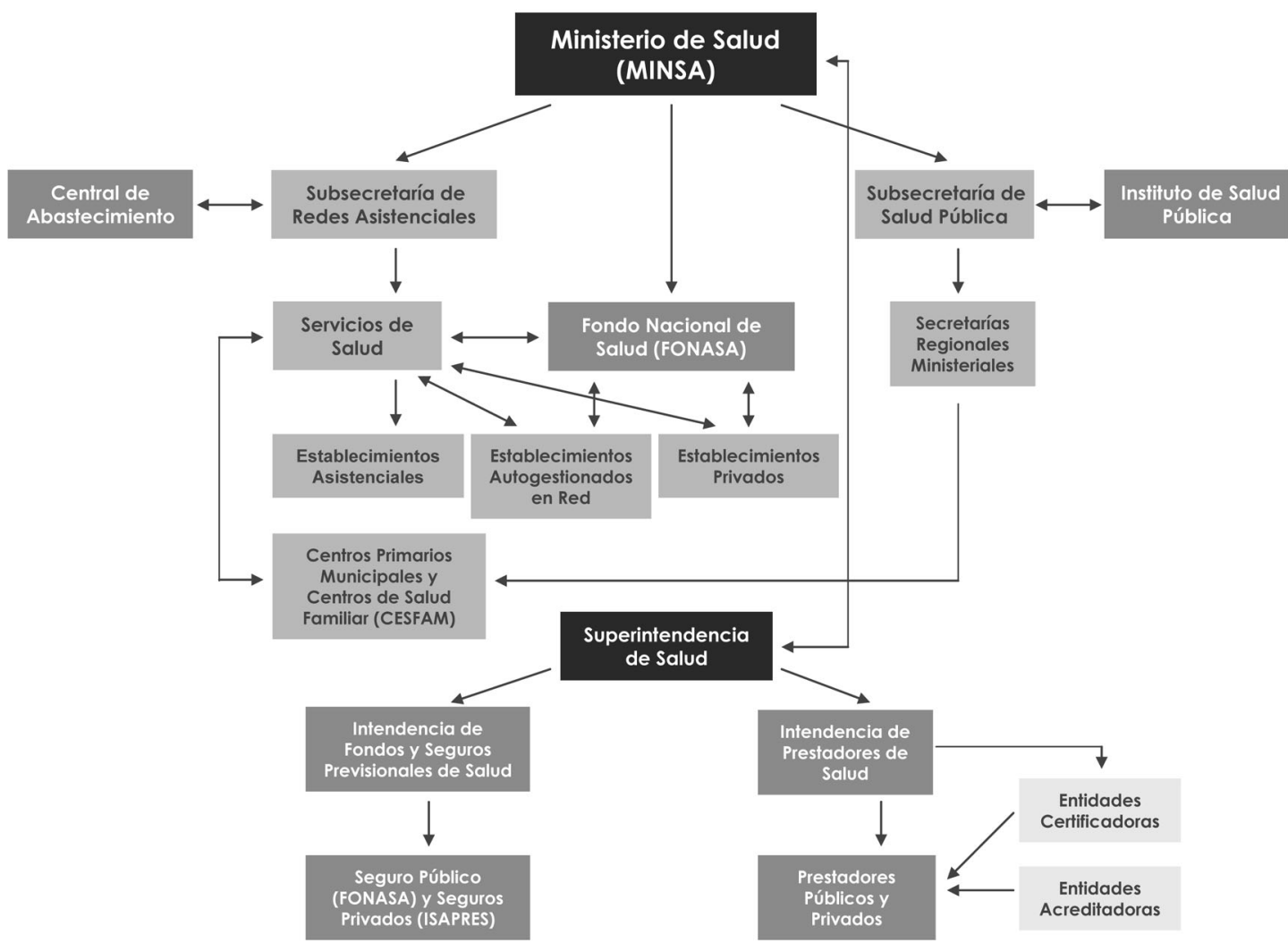

$\longrightarrow$ Dependencia

Relaciones (financiamiento/prestación, otras)

- Ministerio de Salud (MINSA): Rectoría, formulación de las garantías explícitas de salud (GES), fija estándares, establece los sistemas de acreditación y de certificación de especialidades.

- Subsecretaría de Redes Asistenciales: Provisión de servicios de salud, en la modalidad institucional, a la población beneficiaria del sistema público.

- Subsecretaría de Salud Pública: Autoridad sanitaria, vigilancia epidemiológica, fiscalización medio-ambiental y laboral y acciones de salud pública.

- Fondo Nacional de Salud (FONASA): Recaudación, administración y distribución de fondos; compra de prestaciones.

- Central de Abastecimiento: compra de insumos clínicos, materiales y medicamentos y provisión a los establecimientos.

- Instituto de Salud Pública: Laboratorio Nacional y de referencia y control de calidad de los medicamentos.

- Servicios de Salud: Gestión de la red asistencial.

- Secretarías Regionales Ministeriales: Programas de promoción de la salud.

- Superintendencia de Salud: Se relaciona con la Presidencia a través del Ministerio de Salud; controla y supervigila a las Instituciones de Salud Previsional (ISAPRES) y al Fondo Nacional de Salud -beneficios, garantías explícitas de salud (GES)-fiscaliza prestadores públicos y privados en los temas de acreditación, certificación y mantenimiento de estándares).

- Entidades Certificadoras y Acreditadoras: Usan los mismos estándares para prestadores públicos y privados.

Fuente: Elaboración propia.

Por otra parte, se buscó identificar y caracterizar las instancias de articulación diseñadas en los cuerpos legales y reglamentarios.

El análisis documental se complementó con 40 entrevistas en profundidad aplicadas a directivos de salud entre noviembre y diciembre de
2009, a fin de proveer una valoración acerca de si el atributo de la gobernanza sistémica está presente en la institucionalidad a cargo de implementar una política de derechos, así como determinar los factores críticos organizacionales e institucionales que enfrenta la construcción de gobernanza. Se 
incluyó tanto a niveles directivos superiores (Subsecretario de Redes Asistenciales y Superintendente de Salud) como de las redes territoriales de salud y sus establecimientos. Para ello se estableció una muestra trietápica, seleccionando en una primera fase tres regiones del país por su mayor concentración poblacional, dos principalmente urbanas y una con alta presencia rural. Al interior de cada región se seleccionaron al azar dos servicios de salud (SS), atendiendo a que constituyen el nodo clave territorial de las redes asistenciales. En el territorio de la jurisdicción de cada SS, se entrevistaron a los directivos de las redes asistenciales que los SS articulan: en particular, a los directivos de un hospital de alta complejidad y otro de media complejidad; a los de dos Corporaciones o Departamentos de Salud Municipales correspondientes a una comuna con mayor concentración urbana y otra definida como vulnerable por el Ministerio de Salud y dentro de ellas, al azar, a los directivos de un Centro de Salud Familiar (CESFAM), centro de atención ambulatoria del primer nivel definido para operar bajo un modelo de salud familiar. Todos los directivos entrevistados entregaron su consentimiento informado para el desarrollo de la entrevista y de la investigación, el que fue validado por una Comisión Ética en forma previa. Los datos de la entrevista fueron estructurados por dimensiones y procesados con el programa AtlasT1 y contrastados con los hallazgos del análisis documental. Cabe señalar que los datos aquí presentados son parte de resultados más amplios (13).

\section{RESULTADOS}

\section{Las instancias de comunicación y sus efectos sobre la gobernanza}

La revisión del conjunto de normas sobre las instancias formales de articulación de actores que ha originado la reforma, muestra una evidente aspiración a incorporar mecanismos de consulta a partir de consejos, comités y formas similares de distinta composición. Sin embargo, la única instancia creada expresamente para coordinar la operación del sistema público ha sido un Consejo de Integración de la Red
Asistencial (CIRA), órgano de carácter consultivo articulado por el respectivo servicio de salud.

Ahora bien, todos los directores de los servicios de salud entrevistados reconocen la utilidad del CIRA como instancia de articulación de la red asistencial, excepto en un caso donde se manifiesta que ha ido perdiendo el perfil. Lo contrario ocurre cuando los entrevistados son los directores de hospitales cuya opinión es mayoritariamente negativa sea por la tendencia de "programar desde la oferta" o porque se trata de una instancia "simbólica". Los directores de salud municipal mencionan al CIRA como única instancia de salud en la que han participado formalmente en forma posterior a la reforma, pero solo los directivos de los municipios donde el SS ha innovado introduciendo facultades resolutivas al CIRA, reconocen que la instancia es de utilidad. Lo que se valora de aquella instancia de articulación, en el caso de los CESFAM, es la posibilidad de incrementar la resolutividad de tales centros. En la medida en que el CIRA no cumple esa función, su eficacia queda cuestionada.

Por otra parte, Ilama la atención que la reforma no crea instancias intersectoriales que vinculen a los sectores gubernamentales concernidos con la producción de salud. De hecho, la única instancia intersectorial que existe a nivel de las comunas -los Comités Vida Chile- data de los años noventa. Estos Comités buscan conectar a diferentes sectores, pero no forman parte del discurso de los directores de servicios de salud (gestores de la red asistencial). La única mención es que como cada Comité se postula a proyectos, existe una mixtura de proyectos sin ninguna conexión entre sí, lo que redunda en su bajo impacto. Los directores de hospitales prácticamente no aluden a instancias intersectoriales. La valoración cambia cuando los entrevistados son directores de salud municipal (primer nivel de atención) que resaltan la influencia de las instancias intersectoriales en su acción.

Respecto de las tácticas de relacionamiento con otros actores relevantes, se destaca el uso de los territorios definidos por el gobierno regional respectivo para facilitar un acercamiento más focalizado a nivel territorial. De las entrevistas a los directores de hospitales de alta complejidad se desprende el uso del lobby para apalancar iniciativas. Los directores de hospitales comunitarios 
(de baja complejidad) y de CESFAM ponen de relieve el vínculo que se tiene con carabineros, bomberos, el poder judicial y el municipio para extender el abordaje del cumplimiento de las metas sanitarias; todo lo cual da cuenta de la creación de redes pero con un sentido eminentemente instrumental para obtener los incentivos económicos asociados a tales metas.

En conclusión, la dimensión espacial de la gobernanza sistémica, expresada en espacios de comunicación recíproca entre los actores públicos que tienen incidencia en la ejecución de las PPED no aparece como un asunto de consideración expresa de la institucionalidad encargada de la implementación de las PPED en salud. Solo unos pocos actores que han optado por usar las instancias con un sentido más político, incorporando procesos deliberativos y la participación ciudadana, reconocen que han podido desencadenar procesos de producción de significados y saberes compartidos y aumentar la percepción de su interdependencia. Pasemos ahora a examinar los resultados respecto de la dimensión instrumental de la gobernanza.

\section{Los instrumentos de gestión del sector salud en Chile, sus valores, y su incidencia en la gobernanza sistémica}

Un primer hallazgo del análisis documental atinge a la gran variedad de orígenes y naturaleza de los instrumentos de gestión que afectan al tipo de relaciones existentes en el sector salud, más allá de las preestablecidas formalmente.

Destaca la clara preeminencia de los instrumentos transversales externos, particularmente de los indicadores de desempeño y de los Programas de Mejoramiento de la Gestión (PMG) (c), que provienen del Ministerio de Hacienda y que fueron implantados en todo el sector público chileno hace cerca de dos décadas bajo la lógica de la gestión por resultados, tributaria de las reformas institucionales que colocan el énfasis en la asociación entre incentivos económicos, cálculos de beneficios individuales y mediciones. Estos instrumentos comprometen al conjunto de establecimientos públicos de salud aunque son negociados básicamente solo en el nivel central. En general poseen un componente de incentivo monetario para su cumplimiento al que los servicios comprometidos no siempre acceden, como es el caso de algunos PMG. Además, en ellos no existen criterios de valoración del desempeño que aludan a los procesos de gestión del cambio hacia un sistema integrado para la implementación de las PPED. A tales instrumentos se agregan los de naturaleza más política que establecen compromisos en relación a las grandes prioridades gubernamentales y a la creación de instancias de participación ciudadana que deben operar en todo el sector público chileno.

Un segundo cuerpo relevante son los instrumentos transversales internos de gestión del sector salud, referidos a las metas sanitarias y a las GES, estas últimas definidas por ley.

En los espacios territoriales no existen instrumentos formales de articulación entre la Autoridad Sanitaria representada por las Seremis y los servicios de salud. Ello sugiere que el nuevo diseño institucional no facilita la articulación entre las funciones de salud pública y la planificación de la prestación de servicios. Tampoco el diseño institucional contempla instrumentos para la adecuación de las GES y las metas sanitarias en la región. Por otra parte, las relaciones instituidas priorizan el rol de fiscalización del cumplimiento de metas sanitarias generales por sobre la expresión de metas y logros sanitarios a nivel regional pertinentes a cada realidad.

En cuanto a los instrumentos de gestión de las redes asistenciales, los marcos normativos que fundamentan la reforma, actualizan y consolidan las funciones de los servicios de salud e instalan la noción de "gestión de la red asistencial" por sobre la de "dirección de un servicio de salud", lo que incluye la nominación de un director (ahora denominado "gestor de la red") que firma un convenio de "Alta dirección pública" con la Dirección de Servicio Civil (adscrita al Ministerio de Hacienda) cuando gana la posición a través de un concurso público; sin embargo, los convenios, según una crítica ya instalada, aun cuando buscan promover que los directivos enfoquen su atención en el logro de resultados durante su gestión, usan los indicadores de los PMG y, por tanto, no permiten un enfoque en los resultados de la labor directiva. 
Por otra parte, se avanza en el proceso de descentralización al reafirmar la autonomía de los servicios de salud, otorgándoles atribuciones para convenir acuerdos con diferentes prestadores locales de servicios.

Los convenios de gestión entre servicios de salud y municipios buscan restituir su articulación en torno a las metas sanitarias, que incluyen a las GES. La contrapartida de este fenómeno es la virtual ausencia de convenios estructurados entre servicios de salud y hospitales, habida cuenta de que aun cuando la cartera de servicios de cada hospital debe contar con la aprobación del director del servicio de salud correspondiente, no se establecen formalmente metas de producción por tipo de servicios en cada hospital. En el horizonte amenaza a esta fragilidad el advenimiento de los hospitales autogestionados en red, a los que se transfieren facultades administrativas que eran propias de los servicios de salud.

Respecto de los instrumentos de gestión asociados a la salud pública, más allá de los instrumentos de fiscalización ambiental o de programas específicos, las Seremis no disponen de instrumentos de gestión para implantar sus orientaciones al conjunto del sistema regional (prevención, promoción, regulaciones). De hecho, la adherencia a las políticas de salud pública se impulsa básicamente mediante convenios de gestión e incentivos asociados al cumplimiento de metas que provienen de la red asistencial, los cuales no integran el tipo de acciones, competencias y recursos que comporta la gestión de salud pública. Ello limita, particularmente en el nivel primario de atención, el cambio en el eje de las acciones desde lo individual asistencial hacia las acciones colectivas de salud.

Ahora bien, la percepción de los diferentes tipos de actores acerca de la eficacia de los diversos instrumentos para crear gobernanza sistémica, en general confirma tales conclusiones derivadas del análisis de los instrumentos de gestión y permite que afloren nuevas evidencias, como puede apreciarse a continuación.

Los directores de servicios de salud expresan que la enorme diversidad de metas que recaen sobre los servicios, directa o indirectamente, dificulta su desempeño como gestores de red y su enfoque en temas relevantes para el territorio.

La reflexión de los directores de hospitales de alta complejidad deriva permanentemente hacia la problemática de la gestión y sus restricciones, así como a los problemas de recursos. Hay una evidente falta de comprensión de los criterios y mecanismos de transferencia de recursos utilizados por el Fondo Nacional de Salud (asegurador público), al punto que parecen arbitrarios, injustos y no incentivadores para la buena gestión de los hospitales. En paralelo, estos actores señalan que surgen frecuentes requerimientos del Ministerio de Salud de un tipo u otro, fuera de la línea de los convenios de desempeño y no necesariamente consistentes con aquella. Algunos directores explicitan que no existe en el sector un ambiente de negociación, sino más bien de imposición. También se indica que la formulación presupuestaria es vaga a nivel hospitalario y que su ejecución está rodeada de incertidumbre a lo largo del año.

En las entrevistas a los directores de los hospitales comunitarios o de baja complejidad, las GES aparecen como el compromiso más claro, pues se asocia a los bonos de desempeño de los funcionarios, aunque no son objeto de negociación. Lo anterior implica que el sistema hospitalario completo -directivos y agrupaciones de funcionarios- se vuelca al seguimiento y monitoreo del cumplimiento de esas metas. Esto, según lo expresan los actores, puede resultar a veces divergente con las tareas principales que el hospital debe desarrollar para prestar sus servicios.

Las entrevistas a los directores de las corporaciones o departamentos de salud municipales dan cuenta de que el $80 \%$ o $90 \%$ de las acciones programadas de salud de los equipos locales se concentran en dar cumplimiento a las GES, a los programas centrales, a las metas sanitarias y a los Indicadores de Actividad Primaria de Salud (IAPS), dejando un pequeño espacio para atender a necesidades específicas de su realidad local. En municipios pequeños, la adaptación es prácticamente completa, lo que provoca una tensión con la propia readecuación del sistema para atender a las exigencias de implantación del modelo de salud familiar, política nacional débilmente integrada en las metas, según todos los entrevistados.

Los directivos municipales entrevistados señalan, además, que no existe diálogo sobre lo que se debe medir con las metas sanitarias y los IAPS ni datos de fácil acceso y confiabilidad. En general en la negociación sobre las metas prima 
una lógica funcionaria que privilegia la generación de condiciones para obtener los incentivos, y no una lógica que considere la calidad de la gestión de salud en el espacio local. El Plan de Salud Comunal, que se diseña en el primer nivel de atención, es un instrumento desvalorizado, en la medida en que no existe espacio para un despliegue propio de procesos de planificación. Por otra parte, los directores de los CESFAM entrevistados observan un proceso dicotómico, en el cual "se gana" si sus esfuerzos se concentran en el cumplimiento de los compromisos que establecen los instrumentos centrales y "se pierde" si los esfuerzos se sitúan en la reconversión de las acciones hacia el enfoque de salud familiar.

Uno de los máximos responsables del sistema público, resume lo que el propio análisis de los instrumentos de gestión revela como un nudo crítico central, al manifestar que:

...la gestión del cambio a nivel de la red asistencial ha sido insuficiente. Los servicios de salud antes de la reforma eran "totipotenciales o totifuncionales" [...] la reforma les quitó todo el tema de autoridad sanitaria y los transformó básicamente en un holding de entidades prestadoras de salud, algunas de las cuales son parte del servicio y otras que no lo son. Por lo tanto, la gestión de redes es el tema fundamental.

Para apoyar este argumento, entre otros asuntos, sostiene que:

...al revisar las evaluaciones de desempeño que tienen que cumplir los directores de los servicios de salud, y ver cuán alineadas están con la gestión de red, la verdad de las cosas es que en esos compromisos de evaluación de desempeño no se notan.

En conclusión, tanto el análisis documental como los hallazgos de las entrevistas, dan cuenta de una densa red de instrumentos y de una tensión entre las lógicas de los instrumentos transversales externos, cuyo desarrollo es más robusto y su vocación más abarcadora y a la vez más insensible a los requerimientos de integralidad de las PPED (excepto con relación a los indicadores del cumplimiento de las GES) y los instrumentos transversales internos, cuyo desarrollo es más fuerte en los polos Ministerio/servicios de salud (compromisos de gestión), Fondo Nacional de Salud/Subsecretaría de Redes Asistenciales/servicios de salud (contratos de prestación de servicios) y servicios de salud/municipios (Plan de Salud Comunal o Programación en Red), y es mucho más frágil en la relación servicios de salud/hospitales e inexistentes entre servicios de salud/Secretarías Regionales Ministeriales de Salud. En cualquier caso, la preeminencia de instrumentos externos, particularmente de la Dirección de Presupuestos, guiados por una lógica predominantemente sectorial, deviene en que las metas ministeriales y los indicadores de desempeño raras veces son concebidos como "metas integradas" y, por ende, interinstitucionales. Además, las evaluaciones están generalmente asociadas al ejercicio presupuestario cuantitativo anual, más que a logros programáticos-cualitativos y de mediano y largo plazo, como suele ser el caso de transformaciones impactadas por el quehacer sanitario.

En suma, la gestión de la red se ve limitada por dos factores. De una parte, por el peso del instrumental transversal, nucleado por el marco legal de las GES y las metas sanitarias, las que alinean jerárquicamente a las instituciones. De otra parte, por un ajuste institucional inacabado de los instrumentos de gestión propios de la red asistencial para producir mecanismos de coordinación a lo largo de todo el continuo de servicios.

Por otro lado, los entrevistados insinúan que el cúmulo de instrumentos de gestión imponen lógicas y valores que no siempre se condicen con el marco normativo del enfoque de derechos humanos que la actual reforma de la salud pretende impulsar. Claramente, la negociación de las metas (cuando existe) privilegia la generación de condiciones para obtener incentivos económicos y no una lógica que considere los intereses públicos y que refuerce la percepción de las interdependencias.

Por tanto, todo sugiere que también hay una débil consideración de la dimensión instrumental y de la valórica para producir gobernanza sistémica, especialmente si tenemos en cuenta que la literatura reciente ya referida insinúa que la gobernanza está asociada no solo a la contractualización de las relaciones de responsabilidad, sino a la negociación de los compromisos y metas de desempeño, a la existencia de 
incentivos asociados al desempeño que alineen e integren los diversos actores concernidos y a la bidireccionalidad de los sistemas de información.

\section{CONCLUSIONES}

La gobernanza sistémica en el sector salud se ha intentado construir básicamente a partir de instrumentos transversales propios que buscan adaptar el quehacer de las instituciones, desde el nivel central hasta el nivel local (municipios y CESFAM), pasando por los servicios de salud y los hospitales. Ello, según lo reconocen los directores de servicios de salud entrevistados y otras fuentes $(10,30)$, a pesar de las exclusiones del derecho en otros campos, ha tenido efectos en una mayor equidad en la medida en que ha restado arbitrariedad a la elección de las patologías a atender y ha generado responsabilización por la atención, creando nuevas oportunidades de acceso a un conjunto de personas afectadas de enfermedades graves, caras y frecuentes.

En este logro han incidido tres aspectos fundamentales: la consagración de derechos garantizados mediante el cuerpo legal (GES) que genera la obligatoriedad de su cumplimiento; el soporte valórico que ello encuentra en algunos de los directivos de salud; y la adhesión instrumental del personal, asociada a la necesidad del mejoramiento salarial que conllevan los incentivos.

Sin embargo, se evidencian algunos asuntos críticos para la consolidación de las PPED, tanto en sus proyecciones como en su sostenibilidad:

a) La gestión en red, componente sustantivo de la gobernanza sistémica, se zanjó en forma incompleta en la reforma en la medida en que la institucionalidad necesaria para su despliegue, si bien fue contemplada en el espíritu de la ley, no cuenta con las condiciones suficientes (particularmente, de instrumentos e instancias afines a una gestión deliberativa e integrada) para el ejercicio de sus funciones a lo largo del país.

b) La aplicación reglamentaria de la reforma que debía dar cuenta del espíritu del marco legal, consolidó las fracturas de la red sistémica en la medida en que no profundizó en los roles, funciones y atribuciones de las instituciones e instancias que podrían jugar un rol en esa perspectiva.

c) El modelo de asignación de recursos e incentivos destinados al cumplimiento de las metas sanitarias y las GES que alinean a los establecimientos, se tiende a traducir en la postergación del desarrollo de otros componentes de la reforma como el enfoque de salud familiar, el rol de la Autoridad Sanitaria en las regiones y las acciones de salud pública. Tales componentes no quedan adecuadamente integrados en el proceso de responsabilización de los actores municipales y no pueden ser debidamente atendidos por las Seremis, que aparte de la autorización sanitaria, no disponen de suficientes instrumentos para influenciar la red.

d) También juega en contra de la gobernanza sistémica del sector, el cúmulo de instrumentos de gestión externos, muchos de los cuales tienen lógicas y valores que producen tensiones de difícil armonización. En este sentido, no puede soslayarse el hecho de que los ministerios de hacienda imponen reglas de juego no solo en asuntos presupuestarios sino en estándares globales de desempeño.

Bajo este marco, es importante considerar que las metas a veces alinean pero crean incentivos para la competencia destructiva, o sea, no integran. Es evidente, al respecto, que el problema no estriba solo en inhibir los incentivos que contradicen los principios de una PPED, sino en lograr que existan metas orientadas expresamente a la generación de gobernanza.

En términos generales, probablemente una de las lecciones más importantes que el caso de la reforma de la salud en Chile aporta a otras PPED en lo que concierne a la gobernanza sistémica, es que este es un atributo multifacético. Por una parte, supone lograr la alineación de los diversos actores en torno al cumplimiento de las obligaciones emanadas de la consagración del respectivo derecho, cuestión que solo es posible por mandato legal bajo la figura de "órdenes imperativas". Por otra parte, exige que los actores actúen integradamente en un marco de valores que promuevan la comprensión mutua de las interdependencias, la noción de lo público y el respeto a la diversidad. 
Bajo esta perspectiva, lo que sugiere nuestra investigación es que una aceptación acrítica de los modos de gestión y organización heredados de las reformas institucionales acaecidas desde los años '80, puede entrar en contradicción con la necesidad de generar gobernanza dentro del sector salud y, por tanto, atentar contra la integralidad que es particularmente requerida para implantar una PPED. Ello básicamente porque tales reformas establecen una conexión entre incentivos, cálculos privados de beneficios personales y mediciones que no solo no atiende la gestión de las interdependencias, sino porque pueden derivar en un debilitamiento de los valores generales que fun- damentan la ayuda y la cooperación mutua para alcanzar la integralidad.

El desafío en los planos institucional y organizativo parece ser, entonces, conjugar la alineación con la integración, apelando para ello tanto a mandatos legales como a relaciones de responsabilización que creen reglas de juego consistentes con el marco normativo de una PPED y con las especificidades territoriales. No está demás, sin embargo, recordar que los efectos de la parcialidad de los derechos en un marco de recursos escasos, alertan sobre la necesidad de debatir acerca de la inversión y los mecanismos de financiamiento en salud.

\section{AGRADECIMIENTOS}

Este trabajo forma parte del Proyecto FONDECYT 2009 No.1090433 de la Comisión Nacional de Investigación Científica y Tecnológica (CONICYT), Chile, que tiene una duración de dos años (15/03/2009 al 15/03/2011) y se titula "La viabilidad de la institucionalidad pública para la ejecución de políticas con enfoque de derechos. Aproximación desde el caso de las reformas de la salud en Chile". Además de los autores, Eduardo Araya actúa como investigador del proyecto.

Se agradece el apoyo económico brindado por FONDECYT para la realización de la investigación, así como los valiosos comentarios brindados por el Dr. Pedro Crocco con relación a este artículo.

\section{NOTAS FINALES}

a. El sector de la salud en Chile se divide, desde el punto de vista del aseguramiento o protección social, en un sector público y otro privado, ambos parcial o totalmente financiados con aportes que realizan los trabajadores (modelo Bismark) y, en el caso del sector público, con un aporte estatal proveniente de recursos generales de la nación (modelo Beveridge). Estos dos sectores operan con lógicas muy distintas, en atención a que el primero tarifica en función de los riesgos y el segundo los solidariza. La reforma sanitaria se estructuró sobre un conjunto de leyes, que fueron las siguientes, en orden de promulgación: 1) Ley 19.888 de 2003, "de Financiamiento", consideró alzas del impuesto al valor agregado y de otros impuestos específicos para financiar el régimen de Garantías Explícitas de Salud (Plan AUGE) y otros programas; 2) Ley 19.895 de 2003, "Corta de ISAPRES", instruyó sobre normas de solvencia financiera para las Instituciones de
Salud Previsional (ISAPRES) -aseguradores privados ad-hoc en salud- y sobre las transferencias de cartera de afiliados entre éstas; 3) Ley 19.937 de 2004, "de Autoridad Sanitaria", separó las funciones de salud pública de las de prestación de servicios, lo que significó la creación de dos subsecretarías -de Salud Pública y de Redes Asistenciales- y a nivel territorial, el traspaso de las funciones de salud pública desde los servicios de salud a las Secretarías Regionales Ministeriales. Al interior de los servicios de salud, definidos como "gestores de redes", la Ley creó la figura de los Hospitales Autogestionados en Red. La Ley también amplió las facultades de la Superintendencia de ISAPRES asignándole la fiscalización del Fondo Nacional de Salud (asegurador de salud público) y la regulación de los prestadores de salud para la garantía de calidad del Plan de Garantías Explícitas en Salud. 4) Ley 19.966 de 2004, "del Plan AUGE", estableció un plan de salud obligatorio para FONASA e ISAPRES -régimen de Garantías Explícitas en Salud (GES), al principio denominado "AUGE"-, consistente en 
confirmación diagnóstica y tratamientos estandarizados para un conjunto de enfermedades priorizadas por su alto impacto sanitario y social. Este es el "core" de la reforma y su característica clave es la exigibilidad de derechos, en términos de acceso, oportunidad, calidad y cobertura financiera de las prestaciones. 5) Ley 20.015 de 2005, "Larga de ISAPRES", regula la adecuación anual de contratos, alzas de precios y tablas de factores de riesgo por edad y sexo y establece el Fondo de Compensación para las GES, que implica transferencias de recursos entre las ISAPRES, basadas en diferenciales de riesgo de sus carteras de afiliados. La reducción de este fondo a las GES y a su operación solo entre ISAPRES corresponde al componente no logrado de la reforma, según su diseño original, que suponía la creación de un fondo solidario. b. Existe un pequeño porcentaje de las prestaciones de salud otorgadas por las aseguradoras privadas (ISAPRES) que son realizadas por prestadores públicos. Por ejemplo, en el 2008 fue facturado un 2,6\%, haciendo uso de la infraestructura pública tanto en la modalidad de internación hospitalaria como ambulatoria. El total de atenciones ambulatorias del sistema de las ISAPRES realizadas por prestadores públicos fue de $6 \%$, según datos de la Superintendencia de Salud (12).

c. Los PMG buscan el desarrollo de los sistemas transversales de administración (por ejemplo, sistemas de control de gestión, de recursos humanos, etc.) y los asocian a metas vinculadas a beneficios económicos.

\section{REFERENCIAS BIBLIOGRÁFICAS}

1. Organización Panamericana de la Salud. Redes Integradas de Servicios de Salud. Conceptos, opciones de política y hoja de ruta para su implementación en las Américas. Washington DC: OPS; 2010 (Serie La renovación de la Atención Primaria de Salud en las Américas No. 4).

2. Abramovich V, Courtis C. El umbral de la ciudadanía. El significado de los derechos sociales en el Estado social constitucional. Buenos Aires: Editores del Puerto; 2006. (Colección Estudios).

3. Herreño Hernández AL. ¿Todo o nada? Principio de integralidad y derechos sociales. Bogotá: Gente Nueva Editorial; 2008.

4. United Nations. Draft guidelines: a human rights approach to poverty reduction strategies. Ginebra: Office of the High Commissioner for Human Rights; 2002.

5. Comisión Económica para América Latina y el Caribe. La protección social de cara al futuro: acceso, financiamiento y solidaridad. Santiago: CEPAL; 2006.

6. World Health Organization. Towards unity for health: challenges and opportunities for partnership in health development: a working paper. Ginebra: WHO; 2000.

7. Vergara-Iturriaga $M$, Martínez-Gutiérrez $M$. Financiamiento del sistema de salud chileno.
Salud Pública de México [Internet]. 2006 [citado 20 feb 2011];48(6):512-521. Disponible en: http://www.scielo.org.mx/scielo.php?pid = S0036 $-36342006000600010 \&$ script $=$ sci_arttext

8. Urreola R. Restricciones del financiamiento de la salud. En: Fuentes y organización del financiamiento en salud. $4^{\circ}$ Seminario Internacional "Una contribución a la agenda del bicentenario". Santiago de Chile: FONASA, Ministerio de Salud; 2009. p. 137-142 (Ediciones Seminarios Fondo Nacional de Salud Año 3 No.3)

9. Lenz R. Proceso político de la reforma AUGE de salud en Chile: algunas lecciones para América Latina. Una mirada desde la Economía Política. Santiago de Chile: CIEPLAN; 2007. (Serie Estudios Socio/Económicos No. 38).

10. Drago V. La reforma al sistema de salud chileno desde la perspectiva de los derechos humanos. Santiago de Chile: CEPAL; 2006. (Serie Políticas Sociales No. 121).

11. Cunill Grau N. Las políticas con enfoque de derechos y su incidencia en la institucionalidad pública. Revista del CLAD Reforma y Democracia. 2010;46:43-72.

12. Tegtmeier Scherer R. Prestaciones de salud curativas en el Sistema de Isapre. Año 2008 [Internet]. Santiago de Chile: Superintendencia de Salud; 2009 [citado 15 feb 2011]. Disponible en: http://www.supersalud.cl/documentacion/569/articles-5365_recurso_1.pdf 
13. Cunill Grau N, Fernández $M$, Vergara $M$, Araya E. La gobernanza sistémica en el sector salud. Informe del Proyecto FONDECYT No. 1090433. Santiago de Chile: Escuela de Salud Pública, Universidad de Chile; Programa Ciudadanía y Gestión Pública, Universidad de Los Lagos; 2010.

14. Von Haldenwang C. Gobernanza sistémica y desarrollo en América Latina. Revista de la CEPAL. 2005;(85):35-52.

15. Lane C, Bachmann R, editores. Trust within and between organizations. Oxford: University Press; 1998.

16. Innes JE, Booher DE. Collaborative policymaking: governance through dialogue. En: Hajer MA, Wagenaar $\mathrm{H}$, editores. Deliberative policy analysis: understanding governance in the network society. Cambridge: Cambridge University Press; 2003. p. 33-59.

17. Fung A. Survey article: recipes for public spheres: eight institutional design choices and their consequences. The Journal of Political Philosophy. 2003;11(3):338-367.

18. Hajer MA, Wagenaar $H$, editores. Deliberative policy analysis: understanding governance in the network society. Cambridge: Cambridge University Press; 2003.

19. Ranson S, Stewart JD. Management for the public domain. Enabling the learning society. Nueva York: St. Martin's Press; 1994.

20. Sunstein C. Sustituir unos riesgos de salud por otros. En: Elster J, compilador. La democracia deliberativa. Barcelona: Gedisa Editorial; 2001. p. 289-321.

21. Cunill Grau N. La intersectorialidad en la formulación e implementación de la política social [Internet]. CLAD; 2005 [citado 15 feb 2011]. Disponible en: http://www.clad.org/siare_isis/innotend/intersector/info2.html
22. Repetto F. Retos para la coordinación de la política social: los casos de la descentralización y de la intersectorialidad. En: Chiara M, Di Virgilio MM, organizadoras. Gestión de la política social. Conceptos y herramientas. Buenos Aires: Prometeo Libros; 2009. p. 169-200.

23. Agranoff R. Managing within network. Adding value to public organizations. Washington D.C.: Georgetown University Press; 2007.

24. Rhodes RAW. Understanding governance. Policy network, governance, reflexivity and accountability. Buckingham: Open University Press; 1997.

25. Kenis P, Oerlemans L. The social network perspective: understanding the structure of cooperation. En: Cropper S, Ebers M, Hushan C, Smith Ring $\mathrm{P}$, editores. Interorganizational relations. Oxford: University Press; 2008. p. 289-312.

26. Axelsson R, Bihari Axelsson S. Integration and collaboration in public health. A conceptual framework. International Journal of Health Planning and Management. 2006;21(1):75-88.

27. Sen A. ¿Qué impacto puede tener la ética? [Internet]. Congreso Internacional "Los desafíos éticos del desarrollo"; 5-6 sep 2002; Buenos Aires, Argentina [citado 15 feb 2011]. Disponible en: http://participar.org/documentos/Etica_Sen.pdf

28. March J, Olsen J. Democratic governance. Nueva York: The Free Press; 1995.

29. Stivers C. Citizenship ethics in public administration. En: Cooper TL, editor. Handbook of administrative ethics. Nueva York: Marcel Dekker; 2001.

30. Sandoval H. Mejor salud para los chilenos. Cuadernos Médico Sociales. 2004;43(1):5-16.

\section{FORMA DE CITAR}

Cunill Grau N, Fernández MM, Vergara M. Gobernanza sistémica para un enfoque de derechos en salud. Un análisis a partir del caso chileno. Salud Colectiva. 2011;7(1):21-33.

Recibido el 18 de noviembre de 2010

Versión final presentada el 3 de febrero de 2011

Aprobado el 3 de marzo de 2011 